\title{
La práctica docente en áreas STEM: mapeo sistemático de la literatura
}

Esquer Zárate, María Del Pilar; Fernández Morales, Katiuska

La práctica docente en áreas STEM: mapeo sistemático de la literatura

Revista Educación, vol. 45, núm. 1, 2021

Universidad de Costa Rica, Costa Rica

Disponible en: http://www.redalyc.org/articulo.oa?id=44064134038

DOI: https://doi.org/10.15517/revedu.v45i1.42809

\section{(c) $(1) \Theta(9)$}

Esta obra está bajo una Licencia Creative Commons Atribución-NoComercial-SinDerivar 3.0 Internacional. 
Revisiones Bibliográficas

\title{
La práctica docente en áreas STEM: mapeo sistemático de la literatura
}

Teaching STEM: A Systematic Mapping of Literature

María Del Pilar Esquer Zárate

Universidad Autónoma de Baja California, México

pilaresquer01@gmail.com

iD https://orcid.org/0000-0003-3918-3740

Katiuska Fernández Morales

Universidad Autónoma de Baja California, México

katiuska.fernandez@uabc.edu.mx

iD https://orcid.org/0000-0002-6525-2298

\author{
DOI: https://doi.org/10.15517/revedu.v45i1.42809
http://www.redalyc.org/articulo.oa?id=44064134038 \\ Redalyc: http://www.redalyc.org/articulo.oa?id=44064134038
}

Recepción: 22 Julio 2020

Aprobación: 10 Octubre 2020

\section{Resumen:}

La educación y la escolarización se ajustan constantemente a los cambios políticos, sociales, económicos y contextos globales. El impulso de los gobiernos a nivel mundial por los cursos relacionados a las áreas STEM (Ciencia, Tecnología, Ingeniería y Matemáticas, por sus siglas en inglés) ha sido un desafío para las y los educadores, ya que la incorporación e integración de estas áreas en todos los niveles educativos, va fuertemente ligada a la innovación educativa y a las estrategias que el/la docente utiliza en su práctica. Para abonar a este campo del conocimiento el objetivo de esta investigación fue realizar un mapeo sistemático de literatura sobre la producción científica publicada entre los años 2013-2019 que está relacionada con las variables: a) estrategias didácticas, b) herramientas tecnológicas, c) práctica docente, d) STEM y e) innovación educativa, en el marco de la Educación Media Superior y en la Educación Superior. Se plantearon 4 etapas para la realización del mapeo, se utilizaron criterios de inclusión para la búsqueda y se seleccionaron las bases de datos Scopus, Scielo, Google académico, Redalyc, Cambridge collection y Springer. Después de la aplicación de las estrategias de búsqueda se ubicaron un total de 96 artículos. Uno de los hallazgos más importantes en la investigación fue que en los países de Latinoamérica se encontró un mayor número de investigaciones en torno a las variables: estrategias didácticas, herramientas tecnológicas e innovación educativa, mientras que en los países anglosajones se localizaron más investigaciones referentes a la práctica educativa y a las disciplinas STEM.

Palabras ClaVe: Estrategias educativas, Tecnología de la información, Práctica pedagógica, Innovación educacional.

\section{Abstract:}

Education and schooling must constantly adjust to the political, social and economic change of global contexts. Government initiatives worldwide to promote STEM (Science, Technology, Engineering and Mathematics) courses has posed a challenge for educators, since integrating STEM at all educational levels is strongly linked to educational innovation and teaching strategies. The objective of this study is to contribute to this field though a systematic bibliographical review of scientific publications from 2013-2019 related to the following variables: a) Didactic Strategies, b) Technology Tools, c) Teaching Practices d) STEM and e) Educational innovation, within a framework of Higher Secondary Education and Higher Education. Mapping is based on four stages and the inclusion criteria used for the search included the following databases: Scopus, Scielo, Google Academic, Redalyc, the Cambridge Collection and Springer. Selection of the appropriate search provided a result of 96 articles. Among the most significant findings was the larger prevalence of articles about Didactic Strategies, Technology Tools and Educational Innovation in Latin America than the English-speaking countries which had more articles regarding STEM teaching practices.

KEYWORDS: Educational Strategies, Information Technology, Teaching Practice, Educational Innovations.

\section{INTRODUCCIÓN}

La Agenda 2030 para el Desarrollo Sostenible, aprobada en septiembre de 2015 por la Asamblea General de las Naciones Unidas, establece una visión transformadora hacia la sostenibilidad económica, social 
y ambiental de los 193 Estados Miembros de las Naciones Unidas, tal agenda incluye 17 objetivos del Desarrollo Sostenible (ODS), cuyo cuarto hace referencia a una educación de calidad (ODS 4) y tiene como visión "Garantizar una educación inclusiva y equitativa de calidad y promover oportunidades de aprendizaje permanente para todos" (Naciones Unidas, 2019, p.27). En este sentido, la educación y la escolarización se ajustan constantemente a los cambios políticos, sociales, económicos y contextos globales. Además, el impulso de los gobiernos a nivel mundial por los cursos relacionados con las áreas de las ciencias exactas se ha convertido en un desafío para las y los educadores, ya que la incorporación e integración de estas áreas en todos los niveles educativos va fuertemente ligada a la innovación educativa y a las estrategias que el/la docente utiliza en su práctica (Blackley y Howell, 2015).

Hoy en día, el término STEM es usado ampliamente en países como Corea del Sur, Australia, Francia, Gran Bretaña, Alemania, Singapur, Japón, China, Estados Unidos y México. Es importante profundizar el papel que juegan las áreas dentro del término, empezando por la $S$ de ciencia, el cual es un campo que abarca problemas como el calentamiento global, cambios climáticos o áreas de la salud, entre otros. La T de tecnología abarca temas que van desde la construcción del hardware de los dispositivos electrónicos hasta la digitalización a través de la programación (software). La E de ingeniería hace referencia al diseño, infraestructura de edificios, ciudades, etcétera y, por último, la $\mathrm{M}$ de matemáticas que abarca campos que involucran números y ecuaciones (Delgado, 2019).

A partir de este contexto se están creando nuevas industrias y fuentes de empleo, las cuales surgen a medida que avanza la tecnología digital; asimismo, se producen bienes que no se conocían hasta hace algunos años y los procesos productivos son completamente distintos. A los avances en la manufactura se ha sumado la transformación digital, dando paso a la cuarta revolución industrial. Esta realidad exige profesionistas capaces de crear y poner en marcha procesos operativos eficientes, veloces y eficaces, donde el uso de la tecnología es imprescindible. En consecuencia, surge en los Estados Unidos de América el acrónimo STEM, como estrategia para abatir el rezago en la formación de capital humano con talento en la aplicación y uso de las ciencias, la tecnología, la ingeniería y las matemáticas.

Este concepto se ha convertido en un enfoque educativo transdiciplinario e integral que combina el aprendizaje con la aplicación del conocimiento a la vida real y a la resolución de problemas (Alianza para la Promoción de STEM, 2019). Sin embargo, motivar a las y los estudiantes para que se inclinen hacia alguno de estos campos no es cosa sencilla, se requiere la labor del profesorado, es decir, de su práctica en el aula, a partir de la utilización de estrategias didácticas y herramientas tecnológicas que permitan alcanzar los objetivos de aprendizaje de las y los estudiantes e interesarlos en dichas disciplinas.

Antagónicamente, en la práctica docente aún hay evidencia de un rezago importante, las clases de exposición siguen siendo el único medio didáctico y la incorporación de recursos innovadores se reduce solo a sustituir el pizarrón por el cañón y la pantalla (Avendaño, Avendaño, Cruz y Cárdenas, 2014; Barragán, 2016). Por esta razón sigue habiendo investigaciones sobre el desarrollo de programas de capacitación docente, debido a que existe una constante preocupación porque hay un vacío entre el desarrollo de materiales y el uso que el profesorado puede darles en su materia.

Esta necesidad de apoyar e involucrar a las y los profesores en el uso de tecnologías para que produzcan nuevo material didáctico que promueva el trabajo colaborativo y conduzca al alumnado hacia un aprendizaje significativo aún no ha demostrado buenos resultados en las investigaciones realizadas. No hay suficiente información que abunde en los temas de los cambios en los procesos de enseñanza de las y los docentes, quienes aún deben desarrollar la competencia digital que les permita diseñar estrategias innovadoras para la enseñanza y el aprendizaje a través de la tecnología (Bustos y Gómez, 2018). Por otra parte, la falta de uso de herramientas tecnológicas en el aula, en muchas ocasiones, se debe a que el/la docente no tiene acceso a la tecnología necesaria, o en algunos casos, perciben que no es útil para sus asignaturas y no son una prioridad para sus centros educativos (Valdivieso y González, 2016; Zempoalteca, Barragán, González y Guzmán, 2017). 
Según la UNESCO (2016), la innovación educativa implica trascender el conocimiento academicista y transitar del aprendizaje pasivo del estudiantado a un aprendizaje de interacción que se construya entre todos y donde se integren herramientas de apoyo para el trabajo docente, que ayude a descubrir sus potencialidades para innovar y generar cambios en el aprendizaje de las y los estudiantes.

Debido a esto, existe la necesidad de realizar un mapeo sistemático de la literatura, que permita recopilar información acerca de la producción científica existente referente al uso de estrategias didácticas y herramientas tecnológicas que utiliza el profesorado de áreas STEM en su práctica para obtener una visión general de lo que está sucediendo alrededor de la investigación propuesta.

\section{MAPEO SISTEMÁtico DE LA LiTERATURA}

Petticrew y Roberts (2006) sugieren iniciar un trabajo de investigación, realizando revisiones de literatura, ya que proporcionan orientación a las y los investigadores sobre los temas que pretenden investigar a futuro, ofreciéndoles una rica producción científica existente sobre un tema en particular. También señalan que dichas revisiones se pueden utilizar para examinar metódicamente las razones por las cuales una misma pregunta de investigación planteada en diversos estudios arroja conclusiones diferentes.

Una revisión sistemática de la literatura (RSL) es aquella donde a partir de una búsqueda exhaustiva de información acerca de un tema, se analiza detalladamente toda la producción científica disponible para una pregunta de investigación, tema particular o fenómeno de interés. Se basa en una estrategia de búsqueda definida que tiene como objetivo detectar la mayor cantidad de bibliografía relevante para ser analizada de forma minuciosa (Kitchenham y Charters, 2007; García-Peñalvo, 2018).

$\mathrm{Si}$ al iniciar una investigación se descubre que existe poca evidencia empírica sobre el tema en cuestión o que el campo es muy amplio para su análisis, entonces un estudio de mapeo sistémico es un ejercicio apropiado para llevar a cabo antes de la RSL. Esto debido a que permite trazar la evidencia en un dominio con alto nivel de granularidad, porque está diseñado para ofrecer una visión general acerca de una o varias áreas de investigación y para proporcionar información sobre los resultados de otros estudios (Kitchenham y Charters, 2007).

García-Peñalvo (2018) señala que el mapeo se utiliza para complementar a la Revisión Sistemática de la Literatura y afirma que la combinación de ellos asegura mejores resultados en la búsqueda de información. También alude a que dentro de los objetivos de ambos está alcanzar un conocimiento más profundo en un campo particular, ayudan a obtener información sobre tendencias actuales y desafíos posteriores, permiten recopilar fuentes de información sobre los autores más importantes y mencionados, a localizar las revistas con más producción a través de los años y a obtener citas útiles para el desarrollo de investigaciones.

Con base en lo anterior, el objetivo de este trabajo fue realizar un mapeo sistemático de la literatura sobre la producción científica relacionada con las estrategias didácticas y las herramientas tecnológicas enfocadas al área STEM que utilizan las y los profesores en su práctica.

\section{MÉTodo}

Para iniciar con la búsqueda de información, se definieron los pasos metodológicos en el estudio, los cuales se adaptaron de los pasos propuestos por los autores Carrizo y Moller (2018), Navarro y Ramírez (2018) y García-Peñalvo (2018). Se definieron en total cuatro etapas para el proceso del mapeo de la literatura, las cuales se describen a continuación.

Etapa 1. Planteamiento: en esta etapa se contempla la definición de las preguntas de investigación y el objetivo general. 
Etapa 2. Definición de Búsqueda: denominada así, porque involucra las búsquedas piloto y selección de las bases de datos, la definición de los criterios de inclusión y exclusión, la elaboración de las ecuaciones y la búsqueda preliminar de la información.

Etapa 3. Refinamiento de búsqueda y selección de artículos: esta etapa comprende la revisión de artículos seleccionados en la búsqueda preliminar, la elaboración de la lista de cotejo en el Google Drive y el mapeo.

Etapa 4. Análisis de resultados.

\section{Etapa 1. Planteamiento}

En la primera etapa del mapeo sistemático se definieron las preguntas de investigación y el objetivo, los cuales facilitaron el desarrollo de los pasos subsecuentes y posibilitaron una búsqueda amplia de la producción científica existente a nivel mundial sobre las variables de estudio.

Pregunta general: ¿Cuál es la producción científica que ha emergido entre los años 2013 y 2019 sobre las estrategias didácticas, las herramientas tecnológicas y la innovación educativa que utilizan las y los profesores en su práctica en torno a las áreas STEM en la Educación Media Superior y en la Educación Superior?

Preguntas específicas:

P1. ¿En qué año hubo la mayor producción científica sobre las estrategias didácticas, las herramientas tecnológicas y la innovación educativa en torno a las áreas STEM que utilizan las y los docentes en su práctica?

P2. ¿Cuáles son las principales bases de datos donde se recoge información sobre las estrategias didácticas, las herramientas tecnológicas y la innovación educativa en torno a las áreas STEM que utilizan las y los docentes en su práctica?

P3. ¿En qué idiomas se ha producido la mayoría de las investigaciones?

P4. ¿Cuáles son los conceptos emergentes durante el proceso de búsqueda relacionados con estrategias didácticas, herramientas tecnológicas, práctica docente, innovación educativa y STEM?

P5. ¿Cuál es la distribución geográfica donde se está realizando mayor producción científica acerca de las variables de investigación?

Objetivo general: Realizar un mapeo sistemático de literatura sobre las estrategias didácticas, herramientas tecnológicas, práctica docente, STEM e innovación educativa que se implementan en la Educación Media Superior y en la Educación Superior.

\section{Etapa 2. Definición de Búsqueda}

\section{Búsqueda piloto y selección de bases de datos}

Para el desarrollo de esta etapa se comenzó con una búsqueda orgánica para seleccionar las bases de datos que se utilizarían como definitivas en el proceso. La búsqueda se llevó a cabo entre las siguientes bases de datos, las cuales son las más utilizadas en el campo científico:

- Scopus

- Google Académico

- Web of Science

- Redalyc

- Dialnet

- Scielo

- Eric

- Ebsco 
- Cambridge collection

- Springer

Los términos empleados fueron estrategias didácticas, herramientas tecnológicas, práctica docente, STEM, e innovación educativa. Las búsquedas se realizaron en los idiomas inglés y español combinándolos con los caracteres boleanos AND y OR. Se observaron las características principales de cada una de las bases de datos y sus filtros, tal como se muestra en la siguiente tabla (ver Tabla 1):

TABLA 1

Características de las bases de datos

\begin{tabular}{|c|c|c|}
\hline Base de Datos & Principales características & Filtros \\
\hline SCOPUS & $\begin{array}{l}\text { * Ofrece una amplia base de datos de citas y resúmenes de bibliografía } \\
\text { revisada por pares: revistas científicas, libros y actas de conferencias * } \\
\text { Ofrece funciones gratuitas para usuarios no suscritos y está disponible a } \\
\text { través de Scopus Preview * Permite a los usuarios realizar búsquedas } \\
\text { precisas * Proporciona datos actuales y confiables }\end{array}$ & $\begin{array}{l}\text { *Tipo de acceso *Año } \\
\text { de publicación *Tipo } \\
\text { de arbitraje }\end{array}$ \\
\hline $\begin{array}{l}\text { Google } \\
\text { Académico }\end{array}$ & $\begin{array}{l}\text { * Facilita la búsqueda en diversas fuentes desde un solo sitio * Ayuda a } \\
\text { localizar documentos académicos completos * Proporciona } \\
\text { información acerca de documentos académicos claves en un campo de } \\
\text { investigación * Es un buscador especializado en artículos de revistas } \\
\text { científicas, enfocado en el mundo académico * Es una base de datos de } \\
\text { acceso libre y gratuito }\end{array}$ & $\begin{array}{l}\text { "Idioma *Año de } \\
\text { Publicación }\end{array}$ \\
\hline Web of Science & $\begin{array}{l}\text { * Base de datos multidisciplinar de referencias bibliográficas que } \\
\text { proporciona acceso a las colecciones electrónicas * Permite utilizar la } \\
\text { herramienta de análisis para identificar tendencias y pautas }\end{array}$ & *Acceso cerrado \\
\hline Redalyc & $\begin{array}{l}\text { * Integra a su índice las revistas de alta calidad científica y editorial * Base } \\
\text { de datos de acceso libre y gratuito }\end{array}$ & $\begin{array}{l}\text { "Idioma *Año de } \\
\text { Publicación *Área } \\
\text { temática }\end{array}$ \\
\hline Dialnet & $\begin{array}{l}\text { * Base de datos cuyo principal cometido es dar mayor visibilidad a la } \\
\text { literatura científica hispana * Se pueden encontrar fácilmente artículos de } \\
\text { revista, libros y artículos de libros colectivos, actas de congresos, tesis } \\
\text { doctorales, reseñas de otras publicaciones * Base de datos de acceso libre } \\
\text { y gratuito }\end{array}$ & *Tipo de arbitraje \\
\hline ERIC & $\begin{array}{l}\text { Ampliamente utilizada por estudiantes, investigadores/as, miembros de } \\
\text { facultades y otros/as actores que desarrollan informes * Base de datos de } \\
\text { acceso libre y gratuito }\end{array}$ & $\begin{array}{l}\text { "Año de publicación } \\
\text { "Tipo de arbitraje }\end{array}$ \\
\hline EBSCO & $\begin{array}{l}\text { * Brinda a las y los investigadores y estudiantes miles de revistas } \\
\text { académicas a texto completo, asi como acceso a los índices temáticos } \\
\text { principales *Facilita la descarga de archivos * Base de datos de acceso } \\
\text { libre y gratuito }\end{array}$ & "Año de publicación \\
\hline Scielo & $\begin{array}{l}\text { * Acceso internacional a revistas científicas latinoamericanas en ciencias } \\
\text { sociales * Base de datos de acceso libre y gratuito * Pone a disposición del } \\
\text { público textos completos en inglés, sin costo, para aumentar la visibilidad } \\
\text { y accesibilidad de las Ciencias Sociales en América Latina }\end{array}$ & $\begin{array}{l}\text { "País *Idioma *Año } \\
\text { de publicación *Áreas } \\
\text { temáticas *Tipo de } \\
\text { arbitraje }\end{array}$ \\
\hline $\begin{array}{l}\text { Cambridge } \\
\text { collection }\end{array}$ & $\begin{array}{l}\text { * Las listas de publicaciones revisadas por pares comprenden más de } \\
53,000 \text { títulos que cubren investigación académica, desarrollo } \\
\text { profesional, más de } 350 \text { revistas de investigación, educación a nivel } \\
\text { escolar y enseñanza del idioma inglés * Base de datos de acceso libre y } \\
\text { gratuito }\end{array}$ & $\begin{array}{l}\text { *Tipo de acceso *Tipo } \\
\text { de arbitraje *Año de } \\
\text { publicación *Áreas } \\
\text { tematicas }\end{array}$ \\
\hline Springer & $\begin{array}{l}\text { * Ofrece a las y los investigadores de todas las áreas de ciencia, tecnología, } \\
\text { medicina, humanidades y ciencias sociales un lugar para publicar acceso } \\
\text { abierto en revistas y libros * Base de datos de acceso libre y gratuito } \\
\text { (Springeropen) }\end{array}$ & * No tiene \\
\hline
\end{tabular}

Fuente: elaboración propia

Este análisis permitió definir las bases de datos definitivas, se descartó la base Web of Science, ya que al ser de acceso cerrado no se tenían los permisos para utilizarla, Dialnet fue la segunda en eliminarse, ya que no permitía introducir más de 160 caracteres en la barra de búsqueda y, finalmente, se suprimieron ERIC y EBSCO, debido a que no se podía tener acceso a todos los documentos y EBSCO solo permitía guardar los 
resúmenes del texto, lo que complicaría los pasos posteriores del mapeo. Por lo tanto, se eligió trabajar con 6 bases de datos: SCOPUS, Google Académico, Scielo, Redalyc, Cambridge Collection y SpringerOpen.

\section{Criterios de inclusión y exclusión}

Para iniciar con la búsqueda preliminar, se tomaron decisiones en cuanto a las características que ayudarían a determinar qué información sería útil para comenzar, así como las limitaciones pertinentes para decidir qué artículo pasaría a la etapa de refinamiento de búsqueda y selección de trabajos.

Se establecieron los conceptos de búsqueda los cuales fueron: estrategias didácticas, herramientas tecnológicas, práctica docente, STEM e innovación educativa. Se eligieron las seis bases de datos mencionadas en el paso anterior y se precisó el rango de años a utilizar en la búsqueda. Todo esto, con el fin de refinar la estrategia que se seguiría en las búsquedas de información. En la siguiente tabla, se muestran los criterios de inclusión y exclusión establecidos para cada una de las características definidas (ver Tabla 2):

TABLA 2.

Criterios de inclusión y exclusión

\begin{tabular}{|c|c|c|}
\hline Características & Inclusión & Exclusión \\
\hline Arbitraje & Artículos científicos, capítulos de libros, libros & $\begin{array}{l}\text { Artículos de información, tesis de licenciatura, actas de congresos, } \\
\text { ponencias, noticias, blogs especializados, revisiones de libros, tesis } \\
\text { de maestría y tesis de doctorado }\end{array}$ \\
\hline Accesibilidad & Acceso abierto & Acceso cerrado \\
\hline $\begin{array}{l}\text { Periodos de } \\
\text { publicación }\end{array}$ & Publicaciones entre $2013-2019$ & Publicaciones antes del 2013 \\
\hline Nivel Educativo & $\begin{array}{l}\text { Investigaciones enfocadas en la educación media superior y } \\
\text { Superior }\end{array}$ & Investigaciones enfocadas en otros niveles educativos \\
\hline Idiomas & Inglés y español & Otros idiomas diferentes al inglés o al español \\
\hline Palabras clave & $\begin{array}{l}\text { Estrategias didácticas, Herramientas tecnológicas, Innovación } \\
\text { educativa, Práctica docente, STEM }\end{array}$ & No incluye las palabras clave \\
\hline Bases de datos & $\begin{array}{l}\text { Scopus, Scielo, Google Académico, Redalyc, Cambridge collection, } \\
\text { springer }\end{array}$ & Bases de datos diferentes a las seleccionadas \\
\hline
\end{tabular}

Fuente: elaboración propia

\section{Elaboración de ecuaciones de búsqueda}

Para empezar la exploración en las bases de datos, fue necesario definir las ecuaciones de búsqueda, las cuales se formaron con dos o más términos y operadores booleanos, ya que éstos permiten expresar las relaciones que mantienen entre sí para llevar a cabo una búsqueda de información (Ferragud, Vidal, Bertomeu y Lucas, 2017; Ferran y Pérez, 2009).

A continuación, se presenta un extracto de las ecuaciones de búsqueda formadas a partir de las categorías propuestas, las cuales hacían referencia al número de variables por ecuación. A cada ecuación de búsqueda se le asignó un número tal y como se muestra en la Tabla 3. 
TABLA 3

Extracto de ecuaciones de búsqueda por número de variables

\begin{tabular}{ll}
\hline $\begin{array}{l}\text { Número de variable } \\
\text { por ecuación }\end{array}$ & $\begin{array}{l}\text { Número asignado a } \\
\text { cada ecuación } \\
\text { formada }\end{array}$ \\
\hline 1 & $\frac{1}{3}$ \\
\hline 2 & 1 \\
\hline 3 & 5 \\
\hline 5 & 1 \\
\hline
\end{tabular}

Fuente: elaboración propia

Las ecuaciones se clasificaron según el número de variables utilizadas en cada combinación. Como lo muestra la Tabla 3, en el primer ejemplo se tomó como base cada una de las palabras clave con su traducción en el idioma inglés, lo que dio un total de cinco en esta categoría. Para la elaboración de ecuaciones con dos y tres variables, se empleó el diagrama de árbol, que sirve como herramienta para determinar el número de combinaciones posibles entre variables. Esto originó un total de 10 ecuaciones formadas con dos variables y 7 con tres. Finalmente, se formó una con la combinación con todas las variables. Así, se configuraron 23 ecuaciones, las cuales fueron la base para la realización del siguiente paso, que consistió en la búsqueda preliminar de la producción científica bajo los criterios expuestos en la tabla de inclusión.

\section{Büsqueda Preliminar}

El primer acercamiento con los documentos consistió solamente en introducir cada una de las ecuaciones de búsqueda en las bases de datos establecidas en los criterios de inclusión, tomando como filtros: el tipo de arbitraje y el periodo de publicación de los artículos entre 2013 y 2019.

En la Tabla 4, se desglosa el número de artículos encontrados en cada base de datos. La primera columna indica el número de variables por ecuación, y la segunda columna hace referencia al total de ecuaciones formadas por cada combinación entre variables. 
TABLA 4

Cantidad de artículos encontrados por cada ecuación y base de datos

\begin{tabular}{|c|c|c|c|c|c|c|c|}
\hline $\begin{array}{l}\text { Número de variable } \\
\text { por ecuación }\end{array}$ & $\begin{array}{l}\text { Número asignado a } \\
\text { cada ecuación } \\
\text { formada }\end{array}$ & Scopus & Scielo & Google Académico & Redalyc & Cambridge Collection & Springer \\
\hline \multirow[t]{5}{*}{1} & 1 & 189 & 10,165 & 16,700 & 6,745 & 856 & 24 \\
\hline & 2 & 353 & 3,175 & 16,200 & 6,340 & 747 & 34 \\
\hline & 3 & 3,444 & 6,811 & 16,600 & 7,830 & 1,436 & 43 \\
\hline & 4 & 1355 & 314 & 15,900 & 940 & 1,048 & 86 \\
\hline & 5 & 325,909 & 2,534 & $1,300,000$ & 95 & 794 & 7,031 \\
\hline \multirow[t]{10}{*}{2} & 1 & 3 & 290 & 9,960 & 5,485 & 1,520 & 8 \\
\hline & 2 & 0 & 573 & 16,600 & 6,615 & 2,106 & 8 \\
\hline & 3 & 28 & 27 & 10,500 & 750 & 1,744 & 8 \\
\hline & 4 & 3 & 57 & 540 & 85 & 1,539 & 3 \\
\hline & 5 & 15 & 218 & 13,900 & 6,140 & 2,012 & 10 \\
\hline & 6 & 14 & 17 & 9,780 & 845 & 1,651 & 8 \\
\hline & 7 & 14 & 12 & 7,260 & 75 & 1,462 & 2 \\
\hline & 8 & 158 & 34 & 16,200 & 930 & 2,183 & 16 \\
\hline & 9 & 268 & 23 & 16,100 & 95 & 1,996 & 8 \\
\hline & 10 & 70 & 1 & 4,420 & 15 & 1,120 & 22 \\
\hline \multirow[t]{7}{*}{3} & 1 & 1 & 14 & 4,520 & 5,405 & 2,626 & 2 \\
\hline & 2 & 4 & 2 & 3,270 & 800 & 2,287 & 3 \\
\hline & 3 & 0 & 0 & 99 & 70 & 2,138 & 0 \\
\hline & 4 & 1 & 3 & 906 & 75 & 2,531 & 1 \\
\hline & 5 & 1 & 0 & 348 & 15 & 1,718 & 0 \\
\hline & 6 & 7 & 3 & 3,920 & 835 & 2,670 & 3 \\
\hline & 7 & 17 & 0 & 937 & 15 & 2,246 & 3 \\
\hline 4 & 1 & 0 & 0 & 23 & 22 & 3,245 & 0 \\
\hline
\end{tabular}

Fuente: elaboración propia

La búsqueda preliminar arrojó un total de 1929555 artículos de los cuales solo se seleccionaron 1642 . Se descartó la base de datos de Cambridge collection, ya que la cantidad de artículos por ecuación excedía el número definido para el desarrollo de la etapa 3. Entonces, para la selección de documentos, se tomó la decisión de trabajar con los primeros 300 artículos arrojados por cada ecuación de búsqueda. Sin embargo, en el caso de las primeras cinco ecuaciones conformadas por una variable, se trabajó solamente con la base de datos Springer, que arrojaba un total de 187 artículos.

Para las diez ecuaciones que involucraban la combinación de dos variables se eligieron 573 artículos de la base Scopus, 679 artículos de la base Scielo y 93 resultados de Springer. Asimismo, para las siete ecuaciones con la combinación de tres variables, se tomaron 31 archivos de Scopus, 22 de Scielo y 12 de Springer. Finalmente, de la ecuación de búsqueda con todas las variables mezcladas solo se consideró 22 artículos de la base de datos Redalyc y 23 artículos de Google académico, ya que tres bases no arrojaron resultados y la base Cambridge excedía el volumen de artículos establecido por cada ecuación.

\section{Etapa 3. Refinamiento de búsqueda y selección de artículos}

\section{Revisión y selección de artículos encontrados en la búsqueda preliminar}

Antes de iniciar con el desarrollo de este paso se eligió el gestor de referencias Zotero como el indicado para descargar los archivos de manera rápida, ya que es un gestor de acceso abierto que permite recopilar, organizar, citar, sincronizar y acceder a la información disponible en internet. Permite importar datos directamente desde las páginas web visualizadas en el momento, ayuda a generar las citas bibliográficas con los estilos APA, detecta artículos duplicados y permite eliminarlos fácilmente.

La dinámica que se siguió consistió en hacer una primera verificación a los 1642 documentos seleccionados en el primer filtro. La revisión consistió en leer los títulos y los resúmenes de cada texto para identificar las 
palabras clave definidas en los criterios de inclusión. Si los documentos poseían al menos una o varias variables se procedió a descargarlos a través del gestor bibliográfico seleccionado, en el cual se crearon carpetas para cada base de datos.

Con base en lo anterior, se seleccionaron 356 artículos, ya que en 314 de ellos se encontraron una o varias de las palabras clave involucradas. Los 42 documentos restantes se sumaron a la revisión porque, + si bien no contenían exactamente las palabras clave, mencionaban una parte parcial del término o algún sinónimo, razón por la cual se les denominó palabras emergentes. Las más destacadas fueron: estrategias pedagógicas, uso de las tecnologías de información y comunicación (TIC), prácticas pedagógicas, innovación docente, tecnologías digitales, entre otras.

\section{Elaboración de la lista de cotejo para el mapeo}

A continuación, se procedió con la elaboración de una lista de cotejo a través de Google Forms para identificar los elementos que debían poseer los artículos para pasar al siguiente filtro. El objetivo principal de la lista fue recopilar información relevante sobre cada documento, para lo cual se establecieron indicadores que permitían conocer los principales aspectos de divulgación y, además, información acerca del aspecto metodológico de la investigación. Dichos elementos se muestran en la Tabla 5.

TABLA 5

Elementos para el cotejo de artículos en el Google Drive

\begin{tabular}{l}
\hline Aspectos de divulgación de los trabajos de investigación \\
\hline Base de datos \\
\hline Liga del documento \\
\hline Tipo de documento \\
\hline Idioma (inglés o español) \\
\hline Nombre de los autores \\
\hline Producción científica por año \\
\hline Medio de divulgación \\
\hline País \\
\hline Editorial \\
\hline Aspectos metodológicos de los trabajos de investigación \\
\hline Objetivo \\
\hline Pregunta de investigación o problema \\
\hline Método de investigación \\
\hline Participantes en el estudio \\
\hline Instrumentos para la recolección de datos \\
\hline Talidación y confiabilidad del instrumento \\
\hline Nombrias que fundamentaron el estudio \\
\hline Nivel educativo al que iba dirigido el estudio \\
\hline Contiene el concepto de estrategias didácticas \\
\hline Conceptos emergentes relacionados con el concepto estrategias didácticas \\
\hline Contiene el concepto de herramientas tecnológicas \\
\hline Conceptos emergentes relacionados con el concepto herramientas tecnológicas \\
\hline Contiene el concepto de práctica docente \\
\hline Conceptos emergentes relacionados con el concepto práctica docente \\
\hline Conceptos emergentes relacionados con el concepto sTEM \\
\hline Contiene el concepto de innovación educativa \\
\hline Conceptos emergentes relacionados con el concepto innovación educativa \\
\hline
\end{tabular}

Fuente: elaboración propia 
En la sección de aspectos metodológicos de los trabajos de investigación se colocaron indicadores para asegurar si el texto contenía o no elementos que ayudaran a filtrar los documentos, dichos indicadores estaban compuestos por tres valores: una calificación de 5 (si lo posee y lo expone claramente), 3 (lo posee, pero es implícito) y 1 (no lo tiene).

La asignación de valores tuvo como objetivo determinar qué estudios serían los elegidos en la última etapa.

\section{Mapeo}

Después de obtener la base de datos con la información de cada documento resumida, se determinaron los artículos definitivos que se iban a analizar en la Revisión Sistemática de la literatura. Se utilizó la técnica de estadística descriptiva para determinar que artículos del conjunto de datos recogidos, dieran como resultado valores atípicos. La base de datos arrojó una media de 40.4, por lo que se decidió elegir aquellos estudios que hubieran obtenido una calificación igual o mayor al valor obtenido. El resultado de este proceso dio un total de 96 artículos que serán analizados a profundidad en la Revisión Sistemática de Literatura.

\section{Etapa 4. Análisis de resultados}

A partir de la base de datos con la información de recopilada en el mapeo, se pudo dar respuesta a cada una de las preguntas de investigación planteadas al inicio del trabajo.

RP1. ¿En qué año hubo la mayor producción científica sobre las estrategias didácticas, las herramientas tecnológicas y la innovación educativa en torno a las áreas STEM que utilizan las y los docentes en su práctica?

La mayor cantidad de producción científica acerca de los temas en cuestión se identificó en el año 2018 con un total de 45 artículos publicados, seguido por 29 artículos encontrados en 2017, un total de 25 en 2019 y 23 artículos en el año 2016. Entre 2013 y 2015 se encontró la menor cantidad de artículos. La Tabla 6 muestra la producción en el periodo analizado en las bases de datos.

TABLA 6

Producción científica durante los periodos 2013-2019

\begin{tabular}{l|l|l|l|l|l|l|l}
\hline Año & 2013 & 2014 & 2015 & 2016 & 2017 & 2018 & 2019 \\
\hline Porcentaje & $4.7 \%$ & $6 \%$ & $7.4 \%$ & $15.4 \%$ & $19.5 \%$ & $30.2 \%$ & $16.8 \%$ \\
\hline No. Artículos & 7 & 9 & 11 & 23 & 29 & 45 & 25 \\
\hline
\end{tabular}

Fuente: elaboración propia

Se puede observar claramente que en los últimos 4 años se ha producido la mayor cantidad de artículos referidos al uso de estrategias didácticas y herramientas tecnológicas que utilizan las y los docentes en su práctica, así como a la inclusión de la innovación educativa en el aula.

RP2. ¿Cuáles son las principales bases de datos donde se recoge información sobre las estrategias didácticas, las herramientas tecnológicas y la innovación educativa en torno a las áreas STEM que utilizan las y los docentes en su práctica?

De acuerdo con las bases de datos, en Scopus y Scielo, se encontraron los más altos porcentajes de artículos publicados: 72 en Scopus y 46 documentos en Scielo. En la Tabla 7, se muestra el total de artículos obtenidos en cada base de datos. En Redalyc se obtuvieron 17 artículos, en Springer 12 y solo 2 artículos en Google académico. 
TABLA 7

Porcentaje de producción científica por bases de datos

\begin{tabular}{l|l|l|l|l|l}
\hline Base de datos & Scopus & Scielo & Google Académico & Redalyc & Springer \\
\hline Porcentaje & $48.3 \%$ & $30.9 \%$ & $1.3 \%$ & $11.4 \%$ & $8.1 \%$ \\
\hline No. Artículos & 72 & 46 & 2 & 17 & 12 \\
\hline
\end{tabular}

Fuente: elaboración propia

RP3. ¿En qué idiomas se ha producido la mayoría de las investigaciones?

Tal como se observa en la Tabla 8, del total de los 149 artículos que pasaron a la etapa del mapeo, el 50.3\% de los artículos están en el idioma inglés y el $49.7 \%$ en el idioma español.

TABLA 8

Artículos según el idioma

\begin{tabular}{l|l|l}
\hline Idioma & Inglés & Español \\
\hline Porcentaje & $50.3 \%$ & $49.7 \%$ \\
\hline No. Artículos & 75 & 74 \\
\hline
\end{tabular}

Fuente: elaboración propia

P4. ¿Cuáles son los conceptos emergentes durante el proceso de búsqueda relacionados con estrategias didácticas, herramientas tecnológicas, práctica docente, innovación educativa y STEM? Algunos de los conceptos que emergieron en la búsqueda se muestran en la Tabla 9.

TABLA 9

Conceptos emergentes a palabras claves y frecuencias de repetición

\begin{tabular}{lll}
\hline Palabra clave & Conceptos emergentes & Frecuencia \\
\hline \multirow{4}{*}{ Estrategias didácticas } & Estrategias instruccionales & 3 \\
\cline { 2 - 3 } & Estrategias de enseñanza & 2 \\
\cline { 2 - 3 } & Estrategias formativas & 1 \\
\cline { 2 - 3 } & Estrategias pedagógicas & 2 \\
\cline { 2 - 3 } & Estrategias de aprendizaje & 2 \\
\cline { 2 - 3 } & Estrategias docentes & 1 \\
\hline Herramientas tecnológicas & Recursos tecnológicos & 2 \\
\cline { 2 - 3 } & Herramientas digitales & 5 \\
\cline { 2 - 3 } & TIC & 7 \\
\cline { 2 - 3 } & Tecnologías educativas & 1 \\
\hline Práctica docente & Prácticas pedagógicas & 5 \\
\cline { 2 - 3 } & Prácticas educativas & 1 \\
\hline STEM & STEM educativo & 1 \\
\cline { 2 - 3 } Innovación educativa & Innovación instruccional & 1 \\
\cline { 2 - 3 } & Innovación docente & 3 \\
\cline { 2 - 3 } & Innovación pedagógica & 1 \\
\hline
\end{tabular}

Fuente: elaboración propia

Aunque el mapeo sistemático de la literatura ayuda a obtener una visión muy general de un tema en particular, en la Tabla 9 se muestra que las variables inmersas en el presente estudio son llamadas de diversas formas por los diferentes investigadores/as. 
P5. ¿Cuál es la distribución geográfica donde se está realizando mayor producción científica acerca de las variables de investigación?

Como se muestra en la Tabla 10, uno de los hallazgos importantes de este trabajo exhibe que en los países latinoamericanos se encontró un mayor número de investigaciones en torno a los temas de estrategias didácticas, herramientas tecnológicas e innovación educativa, mientras que en los países anglosajones predominan las investigaciones referentes a la práctica educativa y a las disciplinas STEM.

TABLA 10

\begin{tabular}{l|l|l|l|l|l|l}
\hline Continentes & Asia & África & Oceania & Europa & Países Anglosajones & Latinoamérica \\
\hline Porcentaje & $6.04 \%$ & $0.67 \%$ & $1.34 \%$ & $18.8 \%$ & $22.8 \%$ & $50.3 \%$ \\
\hline No. Artículos & 9 & 1 & 2 & 28 & 34 & 75 \\
\hline
\end{tabular}

Fuente: elaboración propia

\section{Discusión}

Uno de los hallazgos más importantes en el desarrollo del mapeo fue que se encontraron diferencias considerables entre el volumen de producción científica generada en distintos países del mundo. En Estados Unidos y Canadá se tiene un mayor impacto en el número de citas referidas a las variables STEM y práctica docente. De los 149 artículos resultantes, 34 abordan la temática en cuestión y fueron desarrollados en Estados Unidos y Canadá. Después de revisar otros trabajos sobre mapeos sistemáticos de la literatura, se identificó que la variable STEM tuvo mayor volumen de producción en Estados Unidos (Ferrada, Díaz, Salgado y Puraivan, 2019); asimismo, en el trabajo en mención, se concluyó que hay evidencias del marcado interés que despierta el término STEM en países altamente desarrollados. Esto quiere decir que los datos entre los dos mapeos realizados son similares, ya que puede observarse que en el país norteamericano se le está dando mayor peso a las investigaciones referentes al campo de la ciencia, tecnología, ingeniería y matemáticas en la educación.

Se encontró que Latinoamérica es el continente donde se realiza un mayor número de contribuciones sobre estrategias didácticas, herramientas tecnológicas e innovación educativa. Ya que un $50.3 \%$ de los artículos, es decir 75 de los 149, corresponden a trabajos relacionados con las variables mencionadas. En contraste, en el mapeo sistemático realizado por Ramírez (2020), se encontró que Estados Unidos es el país donde se ha publicado un mayor número de artículos referentes a innovación educativa y estrategias didácticas. Los hallazgos encontrados a partir de los mapeos y las revisiones pueden diferir, esto puede deberse, en gran medida, a las especificaciones que cada autor toma para el desarrollo de su investigación, es decir, a los criterios de inclusión y exclusión.

Otro hallazgo importante, fue que la mayor cantidad de producción científica acerca de las variables: a) estrategias didácticas, b) herramientas tecnológicas, c) práctica docente, d) STEM y e) innovación educativa se encontraron ubicados en las bases de datos de Scopus y Scielo. De los 149 artículos revisados, un total de 72 fueron publicados en Scopus y 46 documentos en la base de datos Scielo. En otras revisiones sistemáticas, como por ejemplo la que hizo Olvera y Fernández (2020), menciona que las bases de datos donde se encontró mayor volumen de artículos relacionados con estas variables fueron en Web of Science y Redalyc. Esto indica que es posible encontrar información útil en bases de datos de acceso abierto como son Scielo y Redalyc y no exclusivamente en aquellas donde es imprescindible pagar la suscripción. 


\section{Conclusiones}

El mapeo sistemático de la literatura posibilita al lector el establecimiento de rutas propias de exploración acordes a sus propios intereses de investigación, así pues, representa una orientación general en el proceso de selección de la literatura para una revisión más profunda (Navarro y Ramírez, 2018). Por otra parte, la principal fortaleza de la metodología del mapeo es que proporciona evidencia para tener un panorama general, en una forma organizada, sobre el tema a desarrollar en una investigación.

Si bien este proceso se considera una herramienta útil al inicio de una investigación, también tiene que tomarse en cuenta ciertas limitantes que tiene el proceso. Una primera limitante tiene que ver con la pérdida de información valiosa sobre los hallazgos en algunas investigaciones, debido a que, en la etapa de la revisión y selección de los artículos encontrados en la búsqueda preliminar, solo se trabaja con los resúmenes y los títulos de los artículos.

Una segunda limitante es el tiempo dedicado a la elaboración del mapeo, sin embargo, Higgins y Green (2011), comentan que los resúmenes de los artículos identificados a través de una búsqueda bibliográfica pueden ser definidos muy rápidamente para determinar la relevancia potencial que poseen, es decir, a una tasa de lectura estimada de forma conservadora de dos resúmenes por minuto, la revisión de los resultados de una búsqueda en bases de datos podría ser de 120 artículos por hora.

A partir del mapeo sistemático de la literatura realizado en este trabajo se pudo constatar que dicha técnica ofrece una visión general del tema a investigar, ya que facilita el acceso a la producción científica por medio de estrategias de búsqueda que ayudan a seleccionar los documentos susceptibles de un análisis a profundidad a través de la Revisión Sistemática de la Literatura (García-Peñalvo, 2018).

En este mapeo se recopiló información sobre: (a) la producción científica más relevante en los últimos seis años; (b) las bases de datos con mayor número de publicaciones referente a las variables: estrategias didácticas, herramientas tecnológicas, STEM, práctica docente e innovación educativa, y $(\mathrm{c})$ la distribución geográfica de dichas investigaciones, entre otros aspectos.

La importancia de implementar un mapeo sistemático de la literatura al inicio de una investigación se basa en la calidad de la información que puede ser utilizada. Además, metodológicamente hablando, el mapeo proporciona una base de datos ordenados sobre documentos que cumplen con los criterios de calidad seleccionados por el propio investigador/a para realizar las búsquedas y permite seleccionar la información precisa y necesaria para ser analizada a profundidad, optimizando tiempo y proporcionando datos que serán útiles en cualquier etapa de la investigación (Kitchenham y Charters, 2007; García-Peñalvo 2018). En este sentido, a partir del mapeo realizado se pudo observar la evolución cronológica de las variables en cuestión, lo cual permitió observar los aspectos que aún quedan pendientes de análisis para posteriores investigaciones.

Con base en esta investigación se considera importante realizar este tipo de revisiones al inicio de un proyecto, ya que la base de datos que se construye a partir del mapeo brinda información organizada sobre los artículos científicos existentes y útiles, que ayudarán a proporcionar información empírica y relevante sobre el objeto de estudio. El mapeo solo es el primer paso para la Revisión Sistemática de la Literatura, la cual se realizará en un segundo momento y, cuyo resultado es otra base de datos que contiene las problemáticas, marcos teóricos, métodos y principales hallazgos de los artículos revisados, mismos que aportarán información teórica y metodológica al proyecto, ya que se tendrá información útil para la consulta y construcción del trabajo de investigación desde el principio hasta el final.

\section{ReFEREnCias BibliográficAs}

Alianza para la Promoción de STEM (2019).Visión STEM para México. Recuperado de https://movimientostem.o $\mathrm{rg} /$ investigacion/ 
Avendaño B., Avendaño G., Cruz W. y Cárdenas, A. (2014). Guía de referencia para investigadores no expertos en el uso de estadística multivariada. Diversitas: Perspectivas en Psicología, 10(1), 13-27. Recuperado de https://b it.ly/2GseZBU

Barragán, A. (2016). Desarrollo y aplicación de una estrategia didáctica para la integración del conocimiento a la enseñanza de la física en ingeniería. Innovación Educativa, 16(71), 133-155. Recuperado de https://bit.ly/2Gz bkSU

Blackley, S., y Howell, J. (2015). A STEM Narrative: 15 Years in the Making. Australian Journal of Teacher Education, 40(7), 101-112. Recuperado de https://bit.ly/2SlQetC

Bustos, H., y Gómez, M. (2018). La competencia digital en docentes de preparatoria como medio para la innovación educativa. CPU-e. Revista de Investigación Educativa, (26), 66-86. Recuperado de https://bit.ly/2GAIDWs

Carrizo, D., y Moller, C. (2018). Methodological structures of systematic literature review in software engineering: a systematic mapping study. Ingeniare. Revista chilena de ingeniería, 26(Suppl. 1), 45-54. Recuperado de https:// dx.doi.org/10.4067/S0718-33052018000500045

Delgado, P. (2019). Educación STEM: ¿qué es y cómo sacarle provecho? Monterrey, México: Observatorio de Innovación Educativa. Recuperado de https://bit.ly/3ijAfqA

Ferragud, C., Vidal, A., Bertomeu, J. y Lucas, R. (2017). Documentación y metodología en Ciencias de la Salud. Valencia: Nau Llibres. Recuperado de https://bit.ly/33lXhJc

Ferrada, C., Díaz, D., Salgado, N. y Puraivan E. (2019). Análisis bibliométrico sobre educación STEM. Revista Espacios, 40(8), 2-12. Recuperado de https://bit.ly/3ixJfIZ

Ferran, N. y Pérez, M. (2009). Búsqueda y recuperación de la información. Barcelona: UOC. Recuperado de https:// bit.ly/3cVeldf

García-Peñalvo, F. J. (2018). Introducción a las revisiones sistemáticas de literatura. Salamanca, España: Grupo GRIAL. Recuperado de https://bit.ly/2GuldhZ

Higgins, J. y Green, S. (Eds.). (2011). Cochrane Handbook for Systematic Reviews of Interventions Version 5.1.0. The Cochrane Collaboration. Recuperado de http://handbook-5-1.cochrane.org/

Kitchenham, B., y Charters, S. (2007). Guidelines for Performing Systematic Literature Reviews in Software Engineering (EBSE 2007-001). Durham: Keele University and Durham University Joint Report. Recuperado de https://b it.ly/30udF8G

Naciones Unidas (2019). La Agenda 2030 y los Objetivos de Desarrollo Sostenible: una oportunidadpara América Latina y el Caribe. Santiago: CEPAL. Recuperado de https://bit.ly/34emtk4

Navarro, C. y Ramírez, M. (2018). Mapeo sistemático de la literatura sobre evaluación docente (2013-2017).Educaşão e Pesquisa, 44, 1-23. Recuperado de https://bit.ly/3jpIH9f

Olvera, M. y Fernández, K. (2020). Estrategias pedagógicas, herramientas tecnológicas e innovación educativa en la práctica docente en educación superior: mapeo y revisión sistemática de la literatura. Revista innovación educativa (en prensa).

Petticrew, M. y Roberts, H. (2006). Systematic Reviews in the Social Sciences: A Practical Guide. Oxford: Blackwell.

Ramírez, L. (2020). Tendencias de la innovación educativa en los contextos sociales. Análisis del mapeo de literatura. Revista educación, 44(1), 1-27. Recuperado de https://bit.ly/34miphx

UNESCO. (2016). Texto1:Innovación Educativa. Serie "Herramientas de apoyopara el trabajo docente". Perú: Cartolan EIRL. Recuperado de https://bit.ly/2F055Hi

Valdivieso, T. y González, M. (2016). Competencia digital docente: ¿̨ónde estamos? Perfil del docente de educación primaria y secundaria. El caso de Ecuador. Píxel-Bit. Revista de Medios y Educación, (49), 57-63. Recuperado de https://bit.ly/3itMroG

Zempoalteca, B., Barragán, J., González, J. y Guzmán, T. (2017). Formación en TIC y competencia digital en la docencia en instituciones públicas de educación superior. Apertura, 9(1), 80-96. Recuperado de https://bit.ly/ 2GokIsu 